\title{
Die rasante Zerstörung der Urwälder in Südamerika
}

Urs-Peter Stäuble

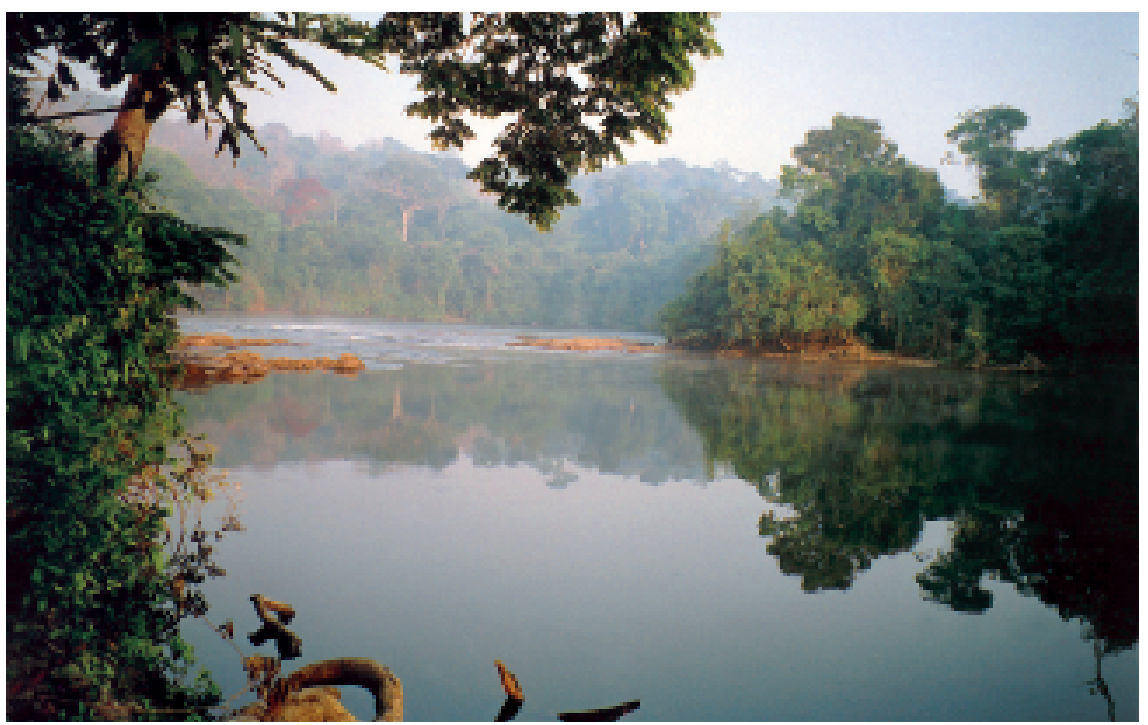

Korrespondenz:

Dr. med. Urs-Peter Stäuble

Brunngrabenstrasse 2

$\mathrm{CH}-4500$ Solothurn

\section{Mittwoch, 17. August 2005}

Mato Grosso, mit 880000 km² die grösste Provinz von Brasilien: Flug in wackeliger Propellermaschine von Cuiabá nach Alta Floresta. Wir sind sehr gespannt, was uns im südlichen Amazonien erwartet. Diese Stadt wurde vor zirka 30 Jahren gegründet, zwischenzeitlich sind ringsum riesige Waldflächen - schätzungsweise zwei Drittel - gerodet und zu Viehweiden sowie zu Soja- bzw. Maisäcker umgewandelt worden. Beim Anflug sehen wir, wie übriggebliebene Urwaldreste vor unseren Augen abgebrannt werden, die Asche zu Haufen aufgeschichtet und verkohlte Baumstämme wie erloschene Zündhölzer daliegen. Es ist zum Weinen, in einer so

kurzen Zeitspanne schafft es unsere abendländische «Zivilisation», eine so wunderbare Artenvielfalt, wie es sie ausschliesslich im Regenwald gibt, zu vernichten.

Ankunft im Hotel Floresta Amazonica: Die Luft riecht nach Rauch und der Himmel ist mit Schwaden verschleiert. Die Sensation ist der nahegelegene Horst einer Harpie (mit $105 \mathrm{~cm}$ der grösste Greifvogel Südamerikas), der einige Monate alte Jungvogel sitzt daneben und beäugt uns mit seiner beeindruckenden Halskrause.
Nachmittags fahren wir mit dem Landrover zum Fluss Teles Pires, mit dem Motorboot geht's weiter den Rio Cristalino flussaufwärts zur gleichnamigen Lodge im Amazonas-Urwald.

\section{Freitag, 19. August 2005}

Mit dem Boot erreichen wir den Anfang des Serra-Trails, eines Pfades, der durch felsiges Gelände auf einen Hügel mit in der Trockenzeit verdorrten Bäumchen führt. Von dort geniessen wir den weiten faszinierenden Ausblick auf den Regenwald und können einige Kleinvögel wie u.a. Tangaren beobachten, die sich an Buntheit übertreffen. Anschliessend kilometerweite Wanderung durch den Urwald mit langen Strecken ohne Vogellaute. Sind solche selten einmal zu hören, hilft meist auch die angestrengteste Suche von vier Augenpaaren nicht, den Vogel zu entdecken, oder falls doch, huscht dieser meist ohne mögliche Bestimmung gleich ins verschluckende Blätterwerk. Am Boden ist es schattig, nur vereinzelt fallen Sonnenstrahlen als Lichttupfer ein. Viele kleine Bäumchen warten darauf, dass einer dieser 50 Meter hohen Riesen umfällt, damit sie ihre Chance wahrhaben können. Wir bewundern stachelbewehrte Palmen, sogenannte "walking palms», die wie auf schrägen Stelzen stehen, und bestaunen die mächtigen Paranussbäume, deren harte kokosnussartige Schale nur von Agutis durchgenagt werden kann.

Zurück auf dem $50 \mathrm{~m}$ hohen schwindelerregenden Beobachtungsturm erfreuen uns ein paar vorbeigleitende Aras.

\section{Mittwoch, 24. August 2005}

Nach dem gestrigen Flug von Cuiabà über São Paulo nach Foz do Iguaçu besuchen wir den Iguaçu-Nationalpark mit seinen weltberühmten Wasserfällen. Diese sind wahrhaftig phantastisch anzuschauen mit diesen vielen breiteren sogenannten Cataratas («del diabolo» der imposanteste) und den Dutzenden von kleineren Wasserfällen von unterschiedlicher Höhe. Wenn man näher rankommt wird's neblig-nass von 


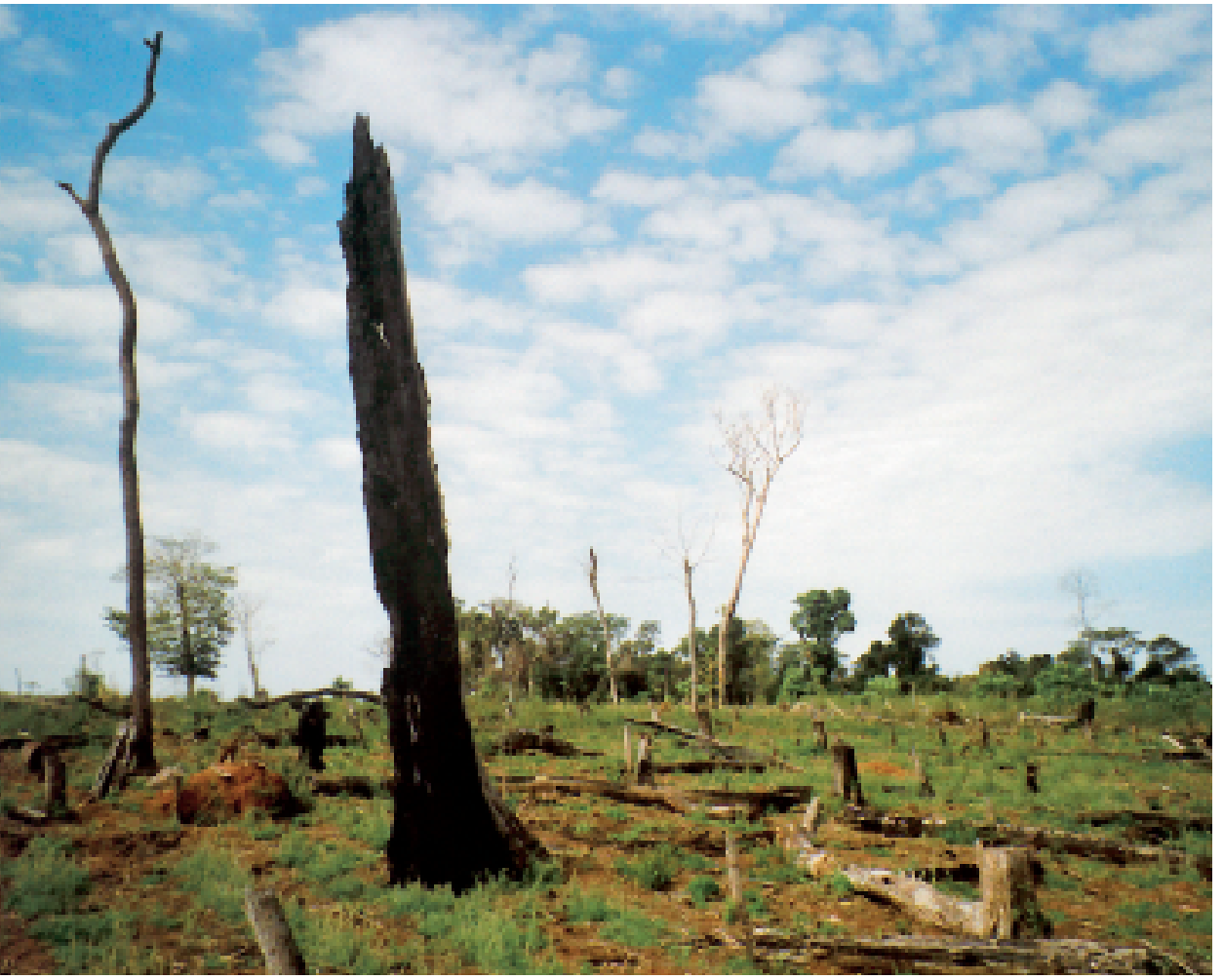

Myriaden von Tropfen und die Sonne macht mehrfache Regenbogen. Die vielen Touristen scheinen von der überwältigenden Pracht fasziniert und fast etwas nervös zu sein. Nachmittags fahren wir über den Fluss Rio Paraná nach Paraguay, die Grenzstadt Ciudad del Este hinterlässt einen deutlich ärmlicheren Eindruck im Vergleich zum benachbarten brasilianischen Foz do Iguaçu. Erst am Abend machen wir uns auf den ungefähr $300 \mathrm{~km}$ weiten Weg zu Christine und Hans Hostettler, die sich vor 27 Jahren nahe der Cordillera San Rafael in der südlichen Provinz Itapúa als Immigranten angesiedelt haben. Nach einer leider nächtlichen Autofahrt werden wir gastfreundlichst bei ihnen empfangen.

\section{Donnerstag, 25. August 2005}

Beide stammen aus Bauernfamilien vom «Gambach» (Schwarzenburgerland/BE) und haben sich hier in harter Arbeit eine abenteuerliche Existenz aufgebaut. Von ungefähr 250 ha Urwald haben sie etwa 150 ha zum Betreiben einer Landwirtschaft gerodet mit Anbau von Soja im Sommer und Getreide im Winter, seit fünf Jahren biologisch. Der ganze Betrieb funktioniert auch dank der Mithilfe von Festangestellten und Taglöhnern bei Bedarf. Christine stellt mir ihre Tätigkeiten für Pro Cordillera San Rafael vor. Das hügelige Gebiet mit $730 \mathrm{~km}^{2}$ atlantischen Urwalds wurde vom Staat zwar zum Nationalpark erklärt, konnte aber wegen fehlender finanziel- ler Mittel den privaten Besitzern nicht abgekauft werden. Der Erwerb von Parzellen zur definitiven Unterschutzstellung sowie die Betreuung des Reservates erfolgt glücklicherweise durch den 1997 von Anwohnern gegründeten Verein Pro Cosara (prcosara@itacom.com.py), wo Christine als Präsidentin die treibende Kraft ist. Ihr Engagement ist enorm und klug ihre Weitsichtigkeit mit Einbezug von staatlichen Stellen, Campesinos, Indios, Schulen und Naturschutzorganisationen (WWF, AVINA ...). Auch hat sie eine Gruppe von sechs Parkwächtern aufgestellt, die täglich zu Kontrollgängen aufbrechen. Hans unterstützt diese mit seinem Ultraleichtflugzeug aus der Luft und kann sie mittels GPS rasch an den Ort des Geschehens dirigieren.

\section{Freitag, 26. August 2005}

Heute machen wir mit dem Landrover eine weite Fahrt von ungefähr $50 \mathrm{~km}$ durch das Reservat auf mit Schlaglöchern durchsetzten Feldwegen zu einem Grundstück, zu dem ich finanziell beigetragen habe. Wir durchqueren diesen aus biologischer Sicht reichhaltigsten subtropischen Wald (Bosque Atlantico), aber auch Campos mit speziell angepassten seltenen Vogelarten. Unterwegs werden mir die Probleme der Bedrohungen des Parks anschaulich vorgeführt. Der Druck der im Wald lebenden Indios und der angrenzenden landlosen Campesinos auf das Reservat ist enorm. Wir sehen frische Brandrodungen, in nächster Nähe zur Parkgrenze steigen auch mehrere Rauchwolken auf. Auf dem mir gezeigten Satellitenbild von 1973 war die ganze Gegend bewaldet, heute beträgt diese Fläche ausserhalb des NP vielleicht noch etwa 10\%. Die Zerstörung geht weiter $\mathrm{zu}$ «Gunsten» von riesigen Monokulturen, die gemäss Hans intensiv chemisch behandelt werden.

Mein Aufenthalt in Paraguay bei Christine und Hans hat sich als der bedeutungsvollste der nun fast vierwöchigen Reise herausgestellt und war ein kurzer, aber sehr wichtiger Abschnitt in meinem Leben. Es war direkter Anschauungsunterricht der rasanten unwiederbringlichen Zerstörung unserer Urwaldlebensräume, aber auch der Kreativität und Leistungsfähigkeit zweier Menschen in einer harmonischen, sich gegenseitig fördernden Partnerschaft zu Gunsten des Naturschutzes.

Liebe Kolleginnen und Kollegen, für eine Spende zugunsten von Pro Cosara möchten wir herzlich danken: Eine Hektare Wald kostet Fr. 250.-, ein Monatslohn für einen Parkwächter Fr. 300.-. Valiant Bank, 3001 Bern, Konto 16 6.102.798.07 6300, zugunsten Pro Cosara Asociación. 